\title{
THE IMPACT OF TARGET VOLUMES OF EHRLICH ASCITES CARCINOMA IRRADIATED WITH A PENCIL SCANNING BEAM OF PROTONS AT A TOTAL DOSE OF 60 Gy ON THE TUMOR GROWTH AND REMOTE EFFECTS IN MICE
}

\author{
T. A. Belyakova ${ }^{1^{*}}$, V. E. Balakin', O. M. Rozanova ${ }^{2}$, E. N. Smirnova ${ }^{2}$, \\ N. S. Strelnikova', A. E. Shemyakov ${ }^{1,2}$, S. S. Sorokina ${ }^{2}$, S. I. Zaichkina ${ }^{2}$
}

${ }^{1}$ Physical Technical Center, Lebedev Physical Institute, Russian Academy of Sciences, Protvino, Moscow oblast, Russia

${ }^{2}$ Institute of Theoretical and Experimental Biophysics, Russian Academy of Sciences, Pushchino, Moscow oblast, Russia

\begin{abstract}
The purpose of the work was to study the growth of solid Ehrlich ascites carcinoma (EAC) and the remote effects (duration of remission, relapse rate, and average lifespan) in tumor-bearing mice exposed to oligofractionated irradiation with the pencil beam scanning of protons (PBSP) at a total dose of 60 Gy depending on the volume of the tissue being irradiated. Experiments were carried out on eight-to nine-week-old SHK male mice. Mice were irradiated with two fractions, $30 \mathrm{~Gy}$ each. In order to determine the volume of irradiated tissue, a tomogram of a mouse in a water phantom was obtained, and a gross tumor volume (GTV) that is equal to the average size of 470 $\mathrm{mm}^{3}$ from all mice was specified using a specially developed $3 D$ planning system. In another group of animals, the irradiated tissue region was increased to the planning target volume (PTV), which was equal to $1500 \mathrm{~mm}^{3}$. An analysis of EAC growth dynamics during the first month showed higher irradiation efficiency in mice with a smaller irradiated volume (the GTV group) compared with the PTV group. In the group with GTV irradiation, survival was higher: the maximum life expectancy in mice without relapse was 5 months longer, and in mice with relapse it was 3 months longer than in the PTV group. The average lifespan $(A L)$ of mice with EAC relapses in the group with GTV irradiation was higher compared to the group with PTV irradiation (96 and 77 days after irradiation or 58 and 31 days after the occurrence of a relapse, respectively; $p \leq 0.01)$. The AL of mice without tumors was also notably longer in the GTV group: 283 days compared to 228 days after PTV irradiation $(p \leq 0.01)$.
\end{abstract}

Keywords: Ehrlich ascites carcinoma, hypofractionation, mice, pencil scanning beam, proton therapy

\section{INTRODUCTION}

Proton therapy (PT) is one of the most developing methods of radiation therapy. It is especially effective in cases when other methods of treatment cannot provide the desired therapeutic effect or could be harmful or dangerous for the patient [1]. The limited use of PT is due to both the high cost of proton accelerators and the scarcity of experimental studies of the biological effects of protons and the ensuing absence of information on the underpinnings of the specific effects of accelerated particles on the key structures and processes in normal and malignant cells $[2,3]$. The parameters of the proton dose distribution are a unique advantage of their physical action: a relatively low entry radiation dose, the presence of a Bragg peak, and the maximum energy deposition at the end of a particle path at the selected depth directly in a tumor. These factors prevent damage to surrounding healthy tissues and organs and lower the probability of side effects. The most promising type of PT is the pencil beam scanning (PBS) technology. It increases the beam-efficiency factor, provides the homogeneity of the dose in a target, and lowers the contribution of secondary particles to the dose $[1,4]$.

Using an electronically guided scanning system and magnets at PBS makes it possible to irradiate a tumor with a thin proton beam. With PBS, the beam position and depth can be controlled, which provides a highly precise delivery of the dose into the tumor.

The application of the pencil beam scanning of protons (PBSP) allows one to decrease the efficient dose to a patient's body by an order of magnitude, which is directly associated with a lower risk of secondary tumors. Consequently, it is clear that further proton beam therapy improvement requires the assessment of hypofractionation or even oligofractionation (one to three fractions) of irradiation, which makes it possible to reduce the duration of treatment and increase its efficiency due to a decrease in the number of fractions and an increase in the single dose. It has been shown that proton irradiation is accompanied by a remarkable decrease in the oxygen enhancement ratio in tissues, minimization of repopulation of tumor cells, suppression of their ability to form metastases, and high efficiency in damaging radioresistant tumor stem cells, which are the cause of the development of secondary tumors in comparison with photon irradiation [5].

The purpose of the work was to study the growth of solid Ehrlich ascites carcinoma (EAC) and the remote effects (duration of remission, relapse rate, and average lifespan (AL)) in tumor-bearing mice exposed to oligofractionated irradiation with a pencil scanning

\footnotetext{
*belyakovatanya@mail.ru
} 
beam of protons at a total dose of 60 Gy depending on the volume of the tissue being irradiated.

\section{MATERIALS AND METHODS}

\subsection{Animals}

The experiments were performed on 8-9-week-old white outbread SHK male mice with a weight of 24-28 g. Animals were maintained in a vivarium of the Institute of Theoretical and Experimental Biophysics, Russian Academy of Sciences (ITEB RAS), under standard conditions and received a dry, standard ration and water ad libitum. Control animals were kept in identical cages and under the same standard conditions as the experimental animals. The shamirradiated control group consisted of 10 animals, and each irradiated group contained 30 animals. The experiments were approved by the ITEB RAS Commission on biological safety and bioethics, and all procedures were carried out in accordance with the regulatory and legal acts concerning the animal experiments and the humane treatment of animals [6].

\subsection{Tumor implantation}

A solid form of EAC was used as a model of tumor growth. This model is a rapidly growing, aggressive, radioresistant, non-metastatic tumor with $100 \%$ death of animals within a month after inoculation of 1-2 million cells [7]. The EAC cell line was obtained from Blokhin National Medical Research Center of Oncology (Moscow, Russia). EAC cells were maintained in vivo in SHK mice by serial intraperitoneal passage at 4-7-day intervals. Ascites developed within 4-6 days after EAC cells inoculation. The ascitic fluid was obtained under aseptic conditions from these mice and washed with phosphate buffered saline three times by centrifugation at $500 \mathrm{~g}$ for $5 \mathrm{~min}$. EAC cells after washing were tested for viability using trypan blue. The cells were counted microscopically using a hemocytometer and suspended in normal saline containing $20^{*} 10^{6}$ cells $/ \mathrm{mL}$. In order to induce the solid form of EAC, mice were injected intramuscularly with $2^{*} 10^{6}$ cells into the hip of the left hind leg.

\subsection{Irradiation of mice}

On the fifth day after inoculation when the tumor node formed in all mice, animals were anesthetized by the intraperitoneal injection of a Xylazine/Zoletil mixture at a dose of $0.7 / 3.4 \mathrm{mg} / \mathrm{kg}$ and fixed on a special platform in such a way that the irradiated limb was maximally distant from the body. Then, the platform was placed in a chamber with warm water (a water phantom) (Fig. 1). Control irradiation of the water phantom with inserted dosimeters instead of the mouse was then performed and a plan of irradiation was prepared.

Irradiation was carried out on a Prometheus proton therapy complex (Physical Technical Center of Lebedev Physical Institute, Russian Academy of Sciences,
Protvino, Russia) with a pencil proton beam from two opposite fields (o and 180 degrees) by scanning the selected tissue volume in the Bragg peak. Proton energy at the output was $87-98 \mathrm{MeV}$ in the first direction and 94-102 MeV in the second. The beam sigma in this energy range at the entrance to the water phantom was $2.8-3.6 \mathrm{~mm}$. Irradiation was performed in a pulse mode (proton pulse duration $200 \mathrm{~ms}$, cycle duration $2 \mathrm{~s}$ ). The dose was controlled by a clinical dosimeter based on a diamond detector (Institute of Physical and Technical Problems, Russia) and radiation monitoring film (Gafchromic EBT2 radiotherapy film, United States).

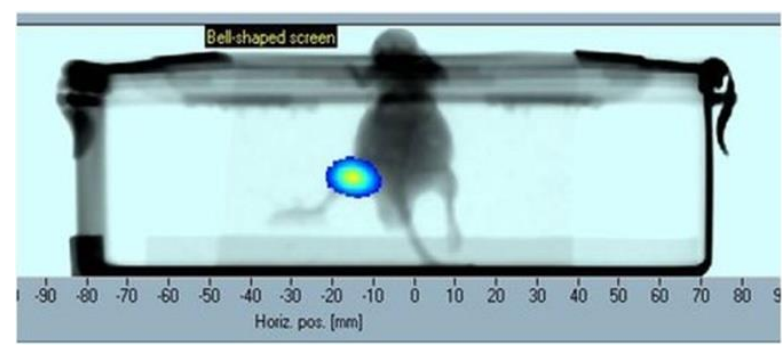

Figure 1. The position of a mouse in the water phantom during irradiation

The dose was calculated in a self-developed treatment planning system, which is a part of the PT complex Prometheus. This program is based on the Monte-Carlo method with dynamic iterative optimization by $\mathrm{min} / \mathrm{max}$ ratio in target volume [8]. In the program, a dose distribution was built with a deviation of the maximum and minimum dose of less than $5 \%$.

To determine the volume of irradiated tissue, conical beam computed tomography of mice in a water phantom was performed, the tumor volume of each mouse was determined, and the average actual tumor volume was calculated to be equal to $470 \pm 25 \mathrm{~mm}^{3}$. The mice were divided into two groups, and the volumes of the irradiated target were chosen: 1) the gross tumor volume (GTV) equal to $470 \mathrm{~mm}^{3}$ and 2) the planning target volume (PTV) equal to $1500 \mathrm{~mm}^{3}$, calculated according to the recommendations of the International Commission on Radiation Units [9]. The GTV is the palpable and instrumentally measured tumor volume, while the PTV includes a clinical volume with an additional space, which is connected with a possible change in the position of a target upon respiration, deviations due to patient positioning, all possible geometric imprecisions, localization and configuration of a tumor, and the peculiarities of irradiation equipment.

In our previous work on the irradiation of solid EAC in mice with two fractions of 30 Gy each by PBSP, it has been shown that the time intervals between fractions from 4 to 24 hours did not affect the antitumor efficiency of the irradiation [10]. In this study, mice were irradiated according to the model of high-dose oligofractionation with two fractions of 30 Gy each with an interval of $24 \mathrm{~h}$. 
In each experiment, there was sham control of mice inoculated with EAC, which took into account the transportation, anesthesia, and simulation of irradiation of animals.

\subsection{Tumor response}

The dynamics of growth of EAC was monitored by measuring the tumor volume once a week within one month with mechanical calipers. The tumor volume $\left(\mathrm{mm}^{3}\right)$ was calculated by the following formula [11]: tumor volume $=\pi / 6^{*} d 1^{*} d 2^{*} d 3$ (where the $d$ values represent three orthogonal diameters).

The response of tumors to treatment was assessed using the antitumor efficiency parameters that characterize the remote effects of radiation: the date of the occurrence of recurrent tumors (remission time), the number of mice with EAC relapse, and the AL of mice with tumors and without them.

\subsection{Statistical analysis}

The statistical assessment of the differences between the groups that have a normal distribution was performed by the Student's $t$-test; in other cases, the nonparametric Mann-Whitney U-test was used.

\section{RESULTS}

The dynamics of the growth of EAC in mice irradiated with a PBSP directed at GTV and PTV tissue volumes is illustrated in Fig. 2. The rate of tumor growth in the irradiated groups was significantly lower than in the control group of unirradiated mice with EAC.

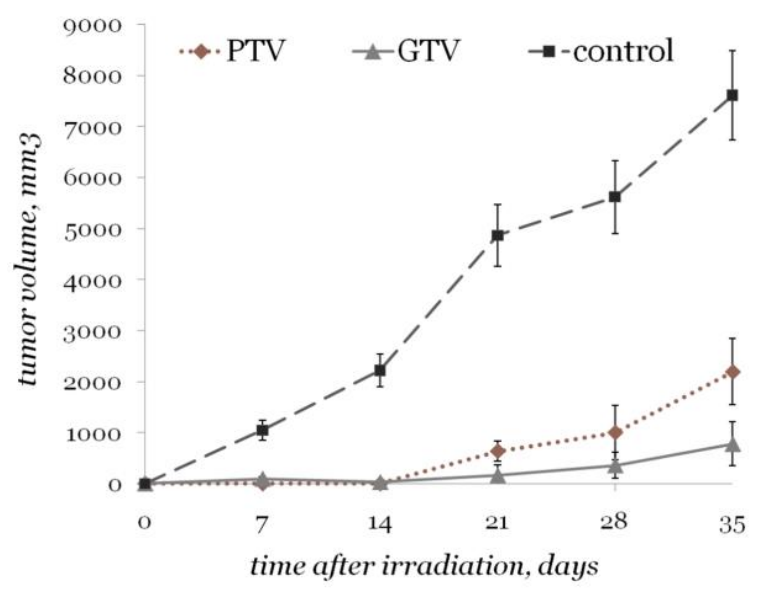

Figure 2. The dynamics of the growth of EAC in mice subjected to GTV and PTV irradiation by a pencil beam of protons with two fractions of $30 \mathrm{~Gy}$. The data are presented as the mean \pm SD. $P \leq 0.01$ vs control

Tumor growth curves do not reveal the number of animals in which the tumor nodules disappear after irradiation; therefore, the number of tumor-free mice was compared at different time points after the irradiation (Table 1). Tumors started to emerge in mice of both groups on day 14 after the irradiation. Comparison of the numbers of tumor-free mice in the groups showed that GTV irradiation was almost twice as efficient as PTV irradiation. The difference made itself evident starting from day 21 after irradiation. The difference became significant by day 35 .

Table 1. The number of tumor-free mice at different times after GTV and PTV irradiation

\begin{tabular}{|c|c|c|c|c|c|}
\hline \multirow{2}{*}{$\begin{array}{c}\text { Irradiated } \\
\text { tissue } \\
\text { volume }\end{array}$} & \multicolumn{5}{|c|}{ Number of tumor-free mice, \% } \\
\cline { 2 - 6 } & $\begin{array}{c}7 \\
\text { days }\end{array}$ & $\begin{array}{c}14 \\
\text { days }\end{array}$ & $\begin{array}{c}21 \\
\text { days }\end{array}$ & $\begin{array}{c}28 \\
\text { days }\end{array}$ & $\begin{array}{c}35 \\
\text { days }\end{array}$ \\
\hline GTV & 99 & 93 & 88 & 81 & 78 \\
\hline PTV & 98 & 82 & 52 & 45 & 37 \\
\hline
\end{tabular}

Analysis of the dynamics of EAC growth within one month showed that the antitumor efficiency in the case of GTV irradiation was higher than after PTV irradiation.

We studied remote radiation effects in mice with a complete regression of tumor nodes after 35 days in PT, i.e., in "cured" animals. At this point, tumors were absent in $78 \%$ of animals exposed to GTV irradiation and $37 \%$ of animals exposed to PTV irradiation.

Further, we studied the dynamics of the EAC recurrence rate after fractionated irradiation of mice with a PSPB of GTV and PTV of the tumors at a total dose of $60 \mathrm{~Gy}$. Irrespective of the irradiated tissue volume, EAC recurrence in the same location was observed within one month after the complete disappearance of the primary tumor in all variants, and the recurrence rates following irradiation of GTV and PTV also did not differ throughout the observation time (Table 2). The growth rates of secondary EACs in both groups did not differ from that of the primary tumor in the unirradiated mice, and the mice died within one month after the appearance of the tumor. The dynamics of the occurrence of relapses in the irradiated groups differed: two months later, the number of mice with relapses was $7 \%$ in GTV group and 33\% in the PTV group. Most of the relapses in the GTV group (53\% from "cured" mice) occurred 3 months after irradiation, and in PTV it remained unchanged (Table 2).

Table 2. The number of mice with relapses at different times after GTV and PTV irradiation

\begin{tabular}{|c|c|c|c|c|c|c|}
\hline \multirow{2}{*}{$\begin{array}{l}\text { irradiated } \\
\text { tissue volume }\end{array}$} & \multicolumn{6}{|c|}{ Number of mice with relapses, \% } \\
\cline { 2 - 8 } & 2 & 3 & 4 & 5 & 6 & 7 \\
\hline GTV & 7 & 53 & 53 & 65 & 65 & 65 \\
\hline PTV & 33 & 33 & 66 & - & - & - \\
\hline
\end{tabular}

Figures 3 and 4 show the dynamics of death of tumor-bearing mice without and with EAC relapses after PT. It can be seen that the viability in the group with GTV irradiation is higher: the maximum lifespan is 5 months longer for mice without relapses and 3 months longer for mice with relapses. The AL of mice with EAC relapses in the group with GTV irradiation was higher compared to the group with PTV irradiation 
(96 and 77 days after irradiation or 58 and 31 days after the occurrence of a relapse, respectively; $p \leq 0.01$ ). The AL of mice without tumors was also notably longer in the GTV group: 283 days compared to 228 days after PTV irradiation $(p \leq 0.01)$. This extreme recurrence indicates that it is not only the preservation of a small number of the most radioresistant tumor cells but also the damage to a large volume of surrounding tissues that play a role in the induction of secondary tumor growth.

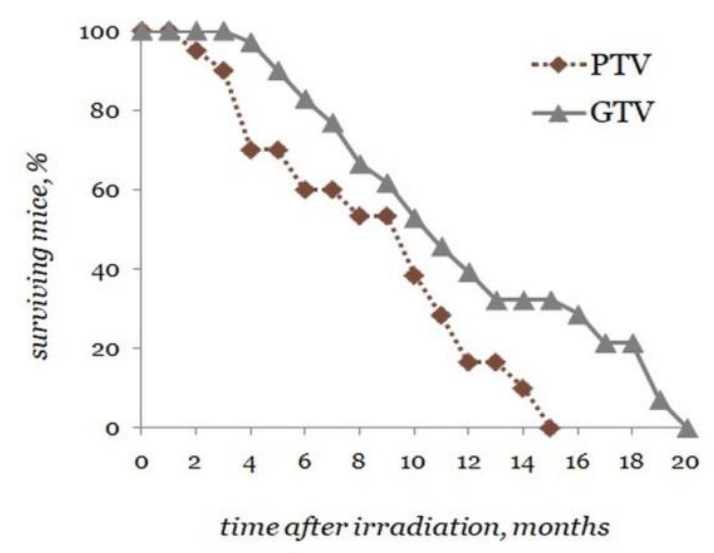

Figure 3. The dynamics of the death of tumor-bearing mice without relapses after proton irradiation of different tissue volumes

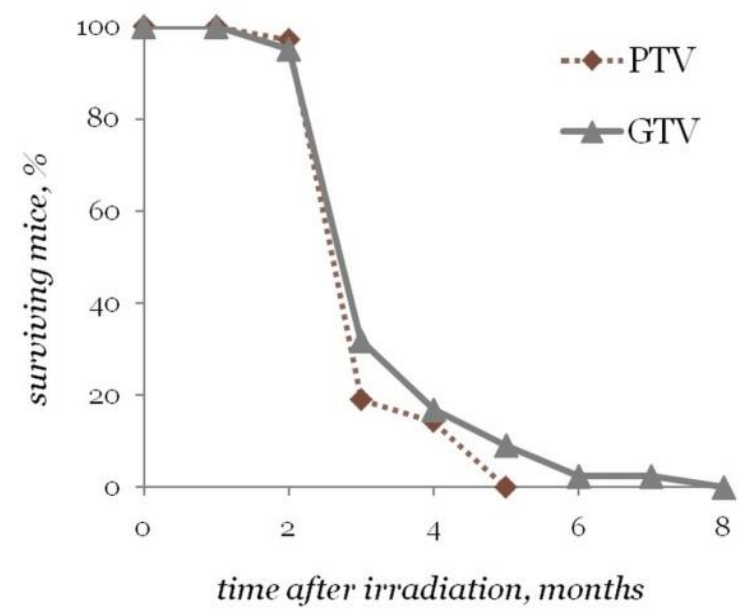

Figure 4. The dynamics of the death of tumor-bearing mice with EAC relapses after proton irradiation of different tissue volumes

\section{DISCUSSION}

The schemes of proton radiotherapy of tumors currently used in clinics are largely similar to those used in conventional radiation therapy that involves exposure to a certain total radiation dose (40-6o Gy in four to eight weeks). The use of these schemes is due to the uncertainty of data on the relative biological efficiency of protons and the low toxicity of the treatment. However, the dose delivered in one episode 26 of irradiation with protons can be increased severalfold relative to the doses used in conventional radiation therapy, since the precise localization of the energy transfer of protons allows minimization of damage to the surrounding tissues. The need for extremely high radiation loads in the treatment of patients with radiation-resistant head and neck tumors, especially squamous cell carcinoma, motivates the search for unconventional approaches to dose fractionation and for adequate experimental models of tumors [12]. No data on the effects of extremely high doses of protons on animals with tumors are currently available. The present study revealed that the antitumor efficiency of irradiation of EAC in mice by PBSP was higher in the case of GTV irradiation than in the case of PTV irradiation with two fractions of $30 \mathrm{~Gy}$ each. An increase in the volume of irradiated tissue to the planned target volume conventionally used in radiotherapy (that is, irradiation of a certain volume of healthy tissue around the tumor) turned out to be less efficient than irradiation of only the actual tumor size. These results open new possibilities for the reduction of the side effects of proton irradiation on adjacent tissues.

We did not find analogous studies of the efficiency of high-dose proton irradiation with relatively long observation time in experimental animal models. Routine schemes of fractionated irradiation with an increase in a single dose with consideration of the relative biological efficiency of protons have been used in few clinical works on remote effects after PT in patients with various cancer types $[5,13]$. The most complete cycle of works carried out on mice irradiated in vivo by accelerated carbon ions in the widened Bragg peak at a single dose of 43.9 Gy focused on the control of the growth of $\mathrm{C}_{3} \mathrm{H} /$ Tif mouse mammary carcinoma implanted into the hip, the frequency and severity of acute dermal reactions (observation for 40 days), and the frequency of radiation fibrosis (observation for 322 days) [11].

The results of our experiments show that the model of solid EAC in mice can be used not only for the study of tumor growth after different proton hypofractionated irradiation regimes but also for the observation of tumor relapses and other remote radiotherapy consequences. Our findings demonstrate a higher antitumor efficiency and a remarkable increase in the AL of tumor-bearing mice after hypofractionated irradiation with PBSP at a total dose of 6o Gy via GTV compared to PTV and contribute to the estimation of immediate risks to surrounding normal tissue and potential late effects.

\section{CONCLUSION}

In summary, the study of the growth of solid EAC and the remote effects (duration of remission, relapse rate, and $\mathrm{AL}$ ) depending on the volume of the tissue in tumor-bearing mice exposed to oligofractionated irradiation with PBSP at a total dose of 60 Gy showed that (1) the antitumor efficiency of irradiation of EAC in mice by a pencil beam of protons in the case of GTV irradiation was higher than in the case of PTV 
irradiation; (2) both PTV and GTV irradiation using the PBSP technology did not affect the rate of EAC relapses and remission time. This may indicate the incomplete death of tumor cells or tumor stem cells; (3) at equal relapse rates in both groups in the variant with GTV irradiation, the AL of mice without tumors substantially increased. These data demonstrate that, for the selected location and size of a solid EAC, increasing the volume of tissue to be irradiated is not necessary to enhance treatment efficiency.

Solid transplantable EAC in mice can be used as an adequate experimental model for the testing of hypofractionated irradiation modes on animals. The tested positioning and cone beam computed tomography of mice with the $3 \mathrm{D}$ planning system and the suggested schemes for hypofractionated irradiation demonstrate the possibilities of the Prometheus proton facility and can be used for further improvement of hadron therapy approaches.

Acknowledgements: The authors are grateful to S.P. Romanchenko for help in conducting the experiments with the animals.

Parts of this work were supported by Grant No. STU151/9-1 from the Physical Technical Center, Lebedev Physical Institute of RAS.

AUTHORS DECLARE THAT THEY HAVE NO COMPETING INTERESTS.

\section{REFERENCES}

1. H. Paganetti, T. Bortfeld, H. Kooy, "Proton Beam Radiotherapy - The State of the Art," Medical Physics, vol. 32, no. 6, pp. 2048-2049, 2005. https://doi.org/10.1118/1.1999671

2. M. Durante, "Proton beam therapy in Europe: more centres need more research," Br J Cancer, vol. 120(8), pp. 777-778, 2019. http://doi.org/10.1038/s41416-018-0329-x PMID: 30531831

3. F. Tommasino, M. Durante, "Proton radiobiology," Cancers, vol. 7, no. 1, pp. 353-381, 2015. http://doi.org/10.3390/cancers7010353 PMID: 25686476

4. M. Mishra, R. Khairnar, S. Bentzen, "Proton beam therapy delivered using pencil beam scanning vs. passive scattering/uniform scanning for localized prostate cancer: Comparative toxicity analysis of PCG 001-09," Clinical and translational radiation oncology, vol. 19, pp. 80-86, Aug. 2019. http://doi.org/10.1016/j.ctro.2019.08.006 PMID: 31650043

5. Е.В. Хмелевский, “Лучевая терапия рака простаты: фотоны, протоны или тяжелые ионы?” Рад. онкол. и ядерная медицина, по. 1, стр. 28-33, 2013. (E.V. Khmelevskii "Prostate cancer radiotherapy: photons, protons or heavy ions?" Radiat. Onkol. Yadern. Med., no. 1, pp. 28-33, 2013.)

6. International Commission on Radiation Units and Measurement, Prescribing, recording, and reporting proton-beam therapy (ICRU Report 78), 2007.

7. S. Mishra, A. Tamta, M. Sarikhani, "Subcutaneous Ehrlich Ascites Carcinoma mice model for studying cancer-induced cardiomyopathy," Scientific reports, vol. 8, no.1, Apr. 2018.

http://doi.org/10.1038/s41598-018-23669-9 PMID: 29618792

8. С.Е. Ульяненко, А.А. Лычагин, С.Н. Корякин, "Распределение дозы и ЛПЭ в биообъектах при облучении протонами," Мед. физика, по.1, стр. 68-74, 2018. (S.E. Ulyanenko, A.A. Lychagin, S.N. Koryakin, "Simulation of dose and LET distributions within biological objects in proton fields," Med. Physics, no. 1, pp. 68-74, 2018).

9. International Commission on Radiation Units and Measurements (ICRU Report 62: Prescribing, Recording and Reporting Photon Beam Therapy), 1999.

10. V.E. Balakin, A.E. Shemyakov, S.I. Zaichkina, "Hypofractionated irradiation of the solid form of ehrlich ascites carcinoma in mice by a thin scanning proton beam," Biophysics, vol. 61, no. 4, pp. 682-686, 2016. http://doi.org/10.1134/Sooo6350916040047

11. B. Sorensen, M. Horsman, J. Alsner, et al., "Relative biological effectiveness of carbon ions for tumor control, acute skin damage and late radiation-induced fibrosis in a mouse model," Acta Oncologica, vol. 54 (9), pp. 1623-1630, 2015. http://doi.org/10.3109/0284186X.2015.1069890 PMID: 26271798.

12. T. van de Water, H. Bijl, C. Schilstra, et al., "The potential benefit of radiotherapy with protons in head and neck cancer with respect to normal tissue sparing: a systematic review of literature," Oncologist, vol. 16(3), pp. 366-377, 2011.

http://doi.org/10.1634/theoncologist.2010-0171 PMID: 21349950

13. S. Rieken, D. Habermehl, T. Haberer, et al., "Proton and carbon ion radiotherapy for primary brain tumors delivered with active raster scanning at the Heidelberg Ion Therapy Center (HIT): early treatment results and study concepts," Radiat Oncol., vol. 7(41), 2012. http://doi.org/10.1186/1748-717X-7-41 PMID: 22436135 